\title{
Practice for determining friction in hydraulic winch systems
}

\author{
G-A. Moslått ${ }^{1}$ M.R. Hansen ${ }^{2}$ \\ ${ }^{1}$ Department of Lifting and Handling, National Oilwell Varco, N-4630 Kristiansand, Norway. \\ E-mail: geir-arne.moslaatt@nov.com \\ ${ }^{2}$ Department of Engineering, University of Agder, 4879 Grimstad, Norway. \\ E-mail: michael.r.hansen@uia.no
}

\begin{abstract}
This paper presents a method for estimating friction in hydraulic active heave compensated (AHC) offshore winches. The method is a two-step approach where the first step is to model the friction loss in the hydraulic motors based on data from the sub-supplier. The second step requires real-life testing, where the remaining friction losses in the winch system is identified and modeled. In this context, a practice is characterized by obtaining a friction loss estimation with the highest possible accuracy over the widest possible range of operating conditions with a limited amount of experimental work. The method benefits from the use of parametric models, sub-supplier data, and real-life measurements on a $150 \mathrm{t}$ AHC crane from National Oilwell Varco Norway (NOVN). The work is an important part of developing a simulation model that can be used actively in virtual testing and verification of crane operations at NOVN. A friction loss model developed from the proposed method was implemented in a NOVN simulation model. Computed and measured hydraulic pressures showed deviations of less than $10 \%$ from measured results for a $150 \mathrm{t}$ crane operating in AHC.
\end{abstract}

Keywords: Hydraulics, torque loss, winch system, offshore knuckle boom cranes.

\section{Introduction}

Offshore heave compensated winches are high-end equipment that is designed to operate under harsh conditions subjected to loads that are not easily reproduced either in laboratories or even during installation. Therefore, there is a huge demand for simulation models that can predict performance under any conditions. The main benefits from having simulation models available for behavior prediction have been more thoroughly described in Moslått et al. (2018) but they may be divided into:

- minimization of equipment costs by using model based design to reduce the level of conservatism in component selection.
- added value to equipment as a behavioral prediction tool useful for planning expensive and complicated operations.

- minimization of testing costs by using virtual testing to reduce level of full-scale testing.

One of the main challenges when predicting behavior is the friction, both in the hydraulic actuation system as well as in the mechanical system. An added challenge is that the winch systems, normally, are tailormade to customers, giving a large amount of different combinations of motors, gear ratios, wire dimensions, drums sizes and wire sheaves. Therefore, a useful behavior prediction requires a modeling technique that handles both the difficulties of setting up friction models of hydraulic-mechanical systems as well as handling 
the use of different components within the same topology. In this paper the focus has been to investigate the winch friction in offshore knuckle boom crane winch systems as the one shown in Fig. 1. An active passive system is divided into two main hydraulic circuits, see Fig. 2. The passive system is designed and controlled to hold the passive weight of the winch load. The passive system is a typical secondary controlled system working under constant pressure connected to an accumulator with a pump that is only used for leakage compensation during operations. The torque needed to hold the passive weight is obtained by use of the variable displacement motors. The active system uses the pump as a primary controller for motion control of the winch. The active system is expected to absorb all remaining loads related to acceleration, load dynamics and friction forces. Friction forces are a significant contributor among these forces and good knowledge and estimates are essential. Experimental validation is crucial for friction modeling, however, it is extremely costly to set up a test scheme that covers the entire operational range of an active-passive winch system. The most common practice today when estimating friction is to use simple constant efficiency factors. This method is based on values provided by sub-suppliers combined with empirical values. This type of modeling has many advantages in that it is simple and easily implemented in early design phases, however, it has obvious limitations in accuracy and does not meet any of the three potential benefits mentioned earlier.

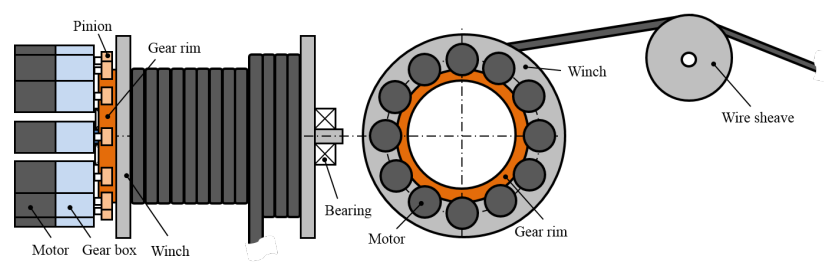

Figure 1: Simple sketch of a winch system.

The friction losses appear in the hydraulic power supply to the hydraulic motors, in each motor, in each gearbox, in each gear-rim connection, in the drum bearings, in the wire and in the sheaves. The friction losses in hydraulic motors have been subjected to research for several decades without any unified recommended practice model appearing. One of the first efficiency models of hydraulic motors were made by Wilson (1948). His model included torque dependent dry friction losses, viscous friction and a constant loss. The model was later used as a base for many other studies with different modifications. Schlösser (1961) modified it in 1961, and added terms including fluid acceleration. In 1969 Jean Thoma included terms to in- clude variable displacement, and the same was done by Pacey et al. (1979). In the following years there were many attempts on improving the loss models and in 1997 Huhtala and Villenius (1997) made a comparison of several models including Wilson, Schlösser, Zarotti, Thoma, Rydberg, Dorey, and their own model. They stated that the accuracy of previous models where inadequate to estimate losses in the full range of hydraulic motors. Huhtatala presented a totally empirical model based on curve fitting. The model was significantly more complex and the details for adapting it to variable displacement motors are unclear. Ortwig came a couple of years later with an in-depth study of several loss mechanisms in the hydraulic motor Ortwig (2002). The study included laminar and turbulent flow losses, pulse losses, churning losses, roller bearing losses, mixed bearing friction, seal losses, valve plate losses and dry friction. He probably saw the downside of having all these terms and made a simpler numerical expression to cover all the loss terms with a similar accuracy. The model was, however, only meant for fixed displacement motors. While Huhtala ended up with a numerical expression, Jeong and Kim (2007) did a similar approach in 2007. But instead of transforming it to a numerical equation, they kept the analytic aspect of the terms and merged them together in one equation. Unfortunately, this work also only considered fixed displacement motors. In 2018 citeMoslatt2018 took the model made by Jeung and Kim, and adapted it for variable displacement and, simultaneously, simplified it. The model showed substantial improvement from the old variable displacement models from Thoma and Pacey, but it also compared favorably with the newer model from Ortwig. The motor type used for that study was axial piston variable displacement motors, with efficiency data given by Bosch Rexroth AG (2010). The hydraulic motors that are easily identified as those

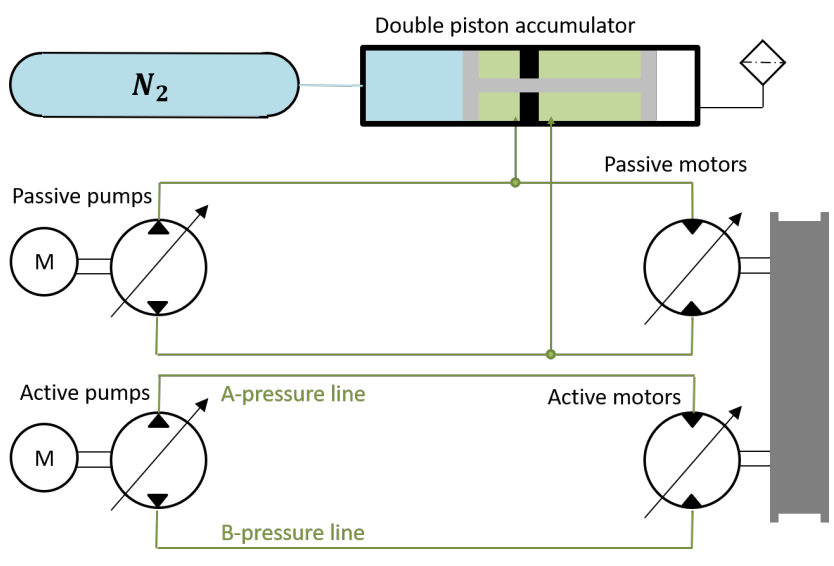

Figure 2: Simplified schematics of an active/passive hydraulic active heave compensated system 
with the most complex friction loss pattern including both mechanical, viscous, and turbulent friction. The idea put forward in this paper is to combine a motor model based on sub supplier data with a model for the friction in the remaining system. The idea is that the friction of the remaining system can be modeled in a simpler way as compared to the motor. The main advantage of this method is that it reduces experimental work while yielding a friction loss model that covers the entire operational range of the winch system.

\section{Method}

The overall method to estimate the friction of a specific hydraulic winch system is carried out in four main steps.

1. Use an established model for axial piston variable displacement motors and obtain the needed parameters for the specific motors in the system.

2. Do friction measurements of the system with several different settings so that measurements capture a wide spectrum of working conditions.

3. Estimate the remaining friction in the system based on the measurements.

4. Use the estimation of the remaining friction to develop a model and specify its parameters.

The steps are described throughout the following sub-sections.

\subsection{Step 1; Model of hydraulic motors}

The sub-suppliers friction data is a table of efficiency values given for a range of operating conditions. There are basically two main ways to use this data. Either using the whole set of data as a map with some kind of interpolation, or fitting a parametric model to represent it. The data given covers most cases but lacks information about low torque, and low speed situations. Also, a parametric model is more easily adapted to situations where the working conditions (mainly fluid viscosity) at the actual plant differs significantly from those of the sub-supplier in-house testing. The parametric model which is mostly based on analytic terms is assumed to be able to cover the blind areas in a better way than an extrapolation of the existing data. This is assumed because the friction-changes in those areas are non-linear and costly to predict with extrapolation. A mapping model compared to a parametric model has the advantage of being simple to generate and will give very accurate results in the range of operating conditions that are given from the supplier. On the other hand the parametric will have a clear advantage when operating outside the operating conditions given by the supplier due to the analytically determined terms. It is also efficient to use and in many cases easier to implement because only an analytical expression is needed instead of a whole map of data. The main downside of having a parametric model is that the parameters will have to be obtained through system parameter identification routines and that a deviation between the desired data and the model have to be expected and accepted.

For parametric friction modeling the model presented in Moslàtt et al. (2018) is chosen. In equation 1 it is assumed that the fluid density and viscosity are constant. Normally, the investigated winch systems have relatively small temperature variations within normal working conditions, however, variations in viscosity at low speed can, potentially, have significant impact on behavior due to mixed lubrication films in the motor. The typical working temperature in the hydraulic oil of the investigated system is in the range of $40^{\circ}$ to $60^{\circ}$ Celsius. This corresponds to a variation in viscosity from $47 \mathrm{cSt}$ to $34 \mathrm{cSt}$ for the standard hydraulic fluid Rando HD46, used by NOVN in these winches.

$$
\begin{array}{r}
T_{f m}=K_{1} \cdot\left|\omega_{m}\right|+K_{2} \cdot \omega_{m}^{2} \cdot D_{m}^{3} \\
+K_{3} \cdot \Delta p_{m} \cdot D_{m}+K_{4}+K_{5} \cdot \Delta p_{m}{ }^{2}
\end{array}
$$

We can identify the $K_{1}$ term as the viscous friction. This type of loss can be found as viscous losses in bearings, churning losses for the cylinder block, viscous slipper friction and viscous friction between barrel and port plate. The $K_{2}$ term is mainly covering the turbulent losses in the inlet port plate. A minor part of the term is covering the churning losses from pistons and slippers although these losses would normally not have the same relationship to displacement setting as the inlet flow loss. The $K_{4}$ term represents the Coulomb friction in motor. The $K_{5}$ term represents mixed lubrication at high pressures. The $K_{3}$ term simply represents a general pressure dependant loss that was introduced to improve correlation between the model and the sub-supplier data.

The model parameters were obtained by means of minimization, using the numerical complex algorithm Box (1965)Krus et al. (1991) with a squared error as cost. The complex method was chosen due to the ease of implementation and that the full code was open and accessible for changes if needed. The cost function considered rotational motor speed from 400 to 2500 $\mathrm{rev} / \mathrm{min}$, displacements from 50 to $D_{m, \max }$, and motor pressure differences of 50 to $350 \mathrm{bar}$.

For the 150 t crane (Fig. 4) investigated in this pa- 


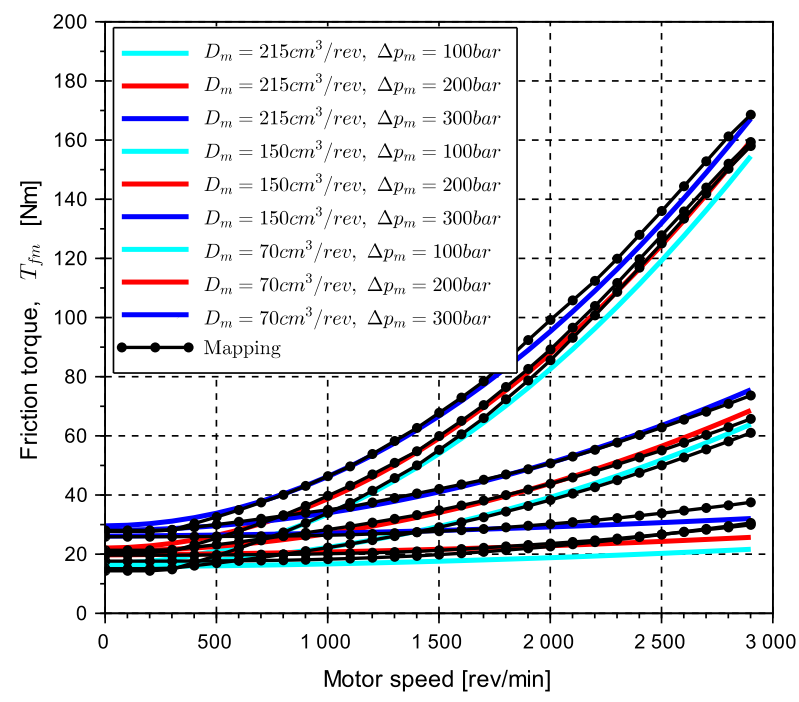

Figure 3: Parametric friction model vs a mapping with linear interpolation of the sub-suplier data for an A6VM (71 series) $215 \mathrm{~cm}^{3} / \mathrm{rev}$ from Bosch Rexroth.

per, the winch is equipped with $11 \mathrm{pcs}$ of $215 \mathrm{~cm}^{3} / \mathrm{rev}$ variable displacement axial piston motors. The friction torque for this motor is shown in Fig. 3. The deviation between mapping and parametric model is overall small, but the deviation is increasing at low displacements and high speeds. To improve confidence in the model parameters, the parameters for a set of three motors were found. The obtained model parameters show a good correlation and some small expected changes dependent on motor size. Following parameters where found for a set of Bosch Rexroth A6VM (71 series) hydraulic motors:

Table 1: Friction model parameters for Bosch Rexroth A6VM hydraulic motor.

\begin{tabular}{|l|l|l|l|}
\hline $\mathbf{D}_{\mathbf{m}, \mathbf{m a x}}$ & $280 \mathrm{~cm}^{3} / \mathrm{rev}$ & $215 \mathrm{~cm}^{3} / \mathrm{rev}$ & $170 \mathrm{~cm}^{3} / \mathrm{rev}$ \\
\hline $\mathbf{K}_{\mathbf{1}}[N m \cdot s]$ & $8.38 \cdot 10^{-3}$ & $4.05 \cdot 10^{-3}$ & $2.48 \cdot 10^{-3}$ \\
\hline $\mathbf{K}_{\mathbf{2}}\left[\frac{N \cdot s^{2}}{m^{8}}\right]$ & $2.58 \cdot 10^{10}$ & $3.69 \cdot 10^{10}$ & $5.01 \cdot 10^{10}$ \\
\hline $\mathbf{K}_{\mathbf{3}}[-]$ & 0.00519 & 0.0050 & 0.0049 \\
\hline $\mathbf{K}_{\mathbf{4}}[N m]$ & 18.1 & 14.0 & 11.0 \\
\hline $\mathbf{K}_{\mathbf{5}}\left[\frac{m^{5}}{N}\right]$ & $1.47 \cdot 10^{-14}$ & $1.16 \cdot 10^{-14}$ & $0.925 \cdot 10^{-14}$ \\
\hline
\end{tabular}

\subsection{Step 2; Full size friction tests}

A full size active heave compensated crane with up to $150 \mathrm{t}$ SWL was tested (Fig. 4).

To obtain the total winch friction, the setup like the one explained in Moslått and Hansen (2018) was

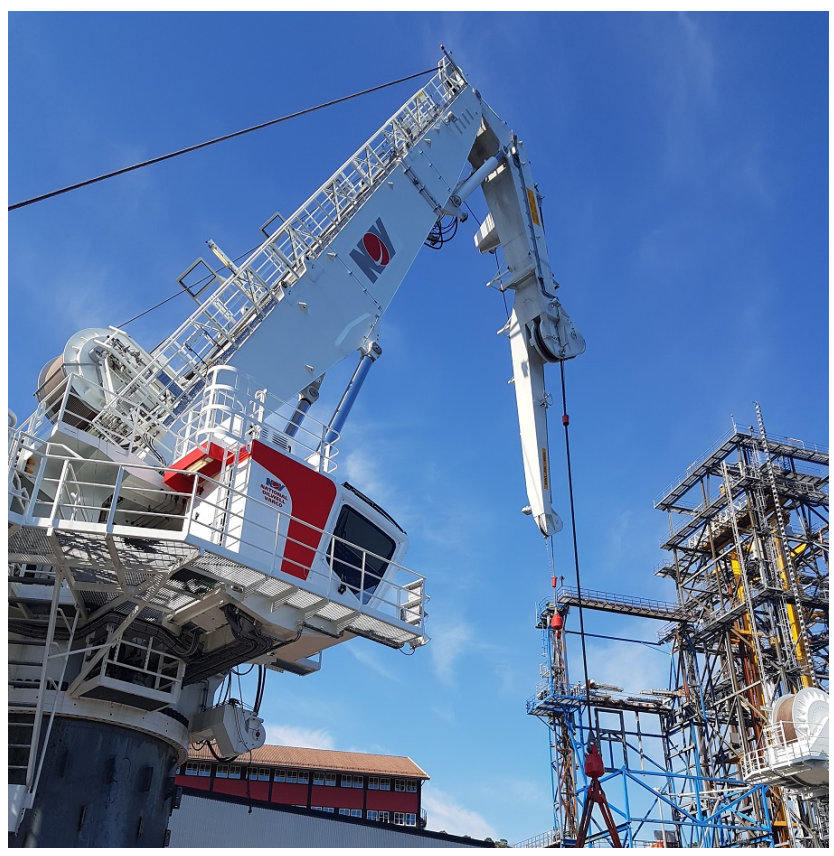

Figure 4: Photo of the crane used for testing.

used. To calculate the winch system losses, the winch is driven at constant speed up and down with an intention to avoid transient behavior. This test is performed with several speeds, loads, and motor displacements variations. For each case, the winch speed, pump displacement, and system pressures are measured. All the data is logged in realtime from the crane PLC. A simplified schematic of the winch system hydraulics that is tested is shown in Fig. 5.

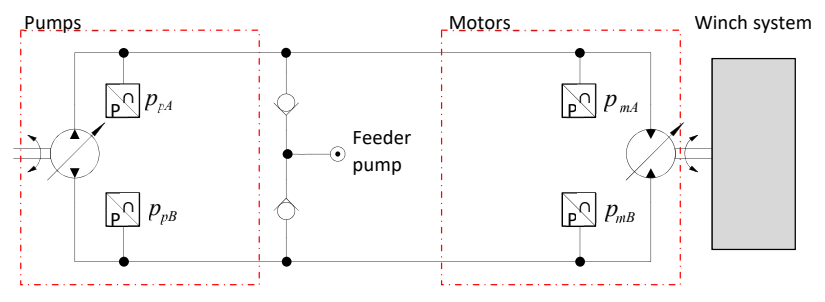

Figure 5: Simplified diagram of the investigated closed loop winch system

In Fig. 6, a typical test sequence is shown that demonstrates that a wide range of the pump capacity is covered.

For every test cycle like this (see Fig. 6), the steady state pressure and winch speed values are retrieved when having constant speed/pump displacement. The values between all these measurement points are estimated based on linear interpolation. The pressure values are used together with the motor displacement 


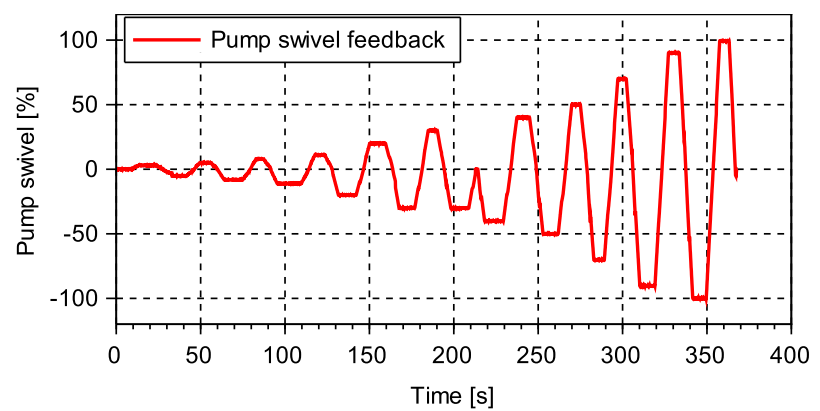

Figure 6: Typical pump displacement for a test sequence used for determining winch friction.

signal to estimate the friction. To calculate the friction loss the first step is to set up the function for pressure difference across the motor $\left(\Delta p_{m}\right)$ when driving up vs down. Note that drag forces on the moving load is neglected since the test is performed with the load in air.

$$
\begin{array}{rlr}
\Delta p_{m} & =p_{m A}-p_{m B} & \\
\Delta p_{m}^{(w u)} & =\Delta p_{L}+\Delta p_{F} & \\
\Delta p_{m}^{(w d)} & =\Delta p_{L}-\Delta p_{F} & \text { (winch up) }
\end{array}
$$

Where $\Delta p_{m}^{(w u)}$ is the pressure drop across the motor when hoisting and $\Delta p_{m}^{(w d)}$ is when lowering. $\Delta p_{F}$ is the total friction losses, and $\Delta p_{L}$ is the pressure difference due to winch load. Calculated friction losses such as pressure (5) is derived from equations (2-4).

$$
\Delta p_{F}=\frac{\Delta p_{m}^{(w u)}-\Delta p_{m}^{(w d)}}{2}
$$

The pressures are measured during the test from transmitter $p_{m A}$ and $p_{m B}$ (see Fig. 5). The friction torque (6) is derived by multiplying with the motor displacement.

$$
T_{f t}=\Delta p_{F} \cdot D_{m}
$$

, where $D_{m}$ is the displacement and $T_{f t}$ is the friction loss referred to as torque on the motor shaft. Each calculation is performed for measurements with the same winch speed. Due to leakage in the hydraulic system, the winch speed for lowering and hoisting will not be the same. Thus the interpolated pressure values are used to be able to compare pressure values at equal winch speed.

The crane operation sequence is shown in Fig. 6 and was performed for multiple load and displacement scenarios. All test scenarios used for friction determination are listed in Table 2

The measured results calculated as friction are seen in Fig. 7.
Table 2: Evaluated test scenarios

\begin{tabular}{l|l|l}
\hline Name & $\begin{array}{l}\text { Motor } \\
\text { displacement } \\
\text { (total/single) }\end{array}$ & Winch load \\
\hline Test 1 & $2365 / 215 \mathrm{~cm}^{3} / \mathrm{rev}$ & $0 \mathrm{t}$ \\
Test 2 & $2365 / 215 \mathrm{~cm}^{3} / \mathrm{rev}$ & $77 \mathrm{t}$ \\
Test 3 & $2365 / 215 \mathrm{~cm}^{3} / \mathrm{rev}$ & $107 \mathrm{t}$ \\
Test 4 & $1800 / 164 \mathrm{~cm}^{3} / \mathrm{rev}$ & $0 \mathrm{t}$ \\
Test 5 & $1800 / 164 \mathrm{~cm}^{3} / \mathrm{rev}$ & $77 \mathrm{t}$ \\
Test 6 & $1800 / 164 \mathrm{~cm}^{3} / \mathrm{rev}$ & $107 \mathrm{t}$ \\
Test 7 & $2200 / 200 \mathrm{~cm}^{3} / \mathrm{rev}$ & $0 \mathrm{t}$ \\
\hline
\end{tabular}

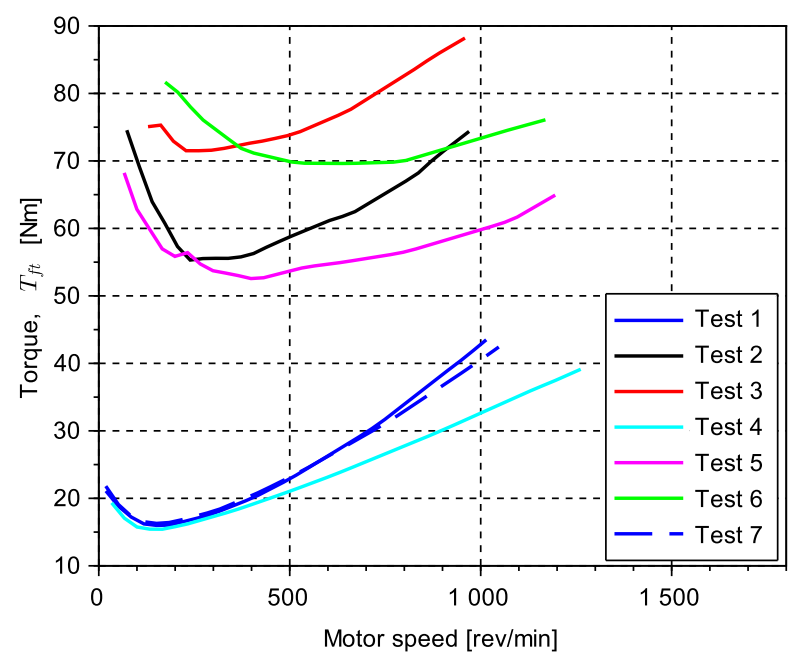

Figure 7: Calculated total winch friction based on measurements. The total torque is divided on 11 motors.

\subsection{Step 3; Estimating remaining system friction}

From the measurements taken, the total winch friction $\left(T_{f t}\right)$ was calculated. The calculations are explained in detail in section 2.2. Based on the results, the motor torque loss is calculated based on the MJ4 model Moslätt et al. (2018). The difference between the calculated MJ4 and the total losses gives the remaining torque loss in the winch system. A model to represent these remaining losses is then implemented, and parameters are identified for optimal results. The remaining system friction is found by subtracting the motor friction from the total measured friction.

$$
T_{f s}=T_{f t}-T_{f m}
$$




\subsection{Step 4; Determine a model for remaining friction}

Based on previous results by Moslàtt and Hansen (2018), equation 8 was chosen to represent the remaining system friction. It includes torque dependent Coulomb and Stribeck terms, and a viscous friction term dependent on winch speed.

$$
\begin{array}{r}
T_{f s}=\left(C_{1} \cdot T_{d}+C_{3}\right) \cdot e^{\frac{-\left|\omega_{m}\right| \cdot 60}{C_{4} \cdot 2 \pi}}+C_{2} \\
+C_{5} \cdot \omega_{m}+C_{6} \cdot T_{d}
\end{array}
$$

The model has a Stribeck shape to describe the dry friction at $0 \mathrm{rev} / \mathrm{min}$ and the exponential curve to account for any mixed lubrication at low speed, a Coulomb and viscous friction term. The first and second are load-dependent, and the third depends on the rotational speed of the drives.

The parameters $C_{1-6}$ were optimized based on test data from the crane. The data used to optimize the model parameters are the tests mentioned in table 2 . The parameters were identified by means of minimization of the deviation between measured and modeled friction. All seven test cycles were considered during the optimization (test $=1,2 . .7$ ). For every test the error was calculated over the whole range of measured velocities (from $i_{\min }$ to $i_{\max }$ ) with an iteration step of 25 $\mathrm{rev} / \mathrm{min}$. The total cost, E, was calculated according to $(9)$.

$$
E=\sum_{t e s t=1}^{7} \sum_{i=i_{\min }}^{i_{\max }}\left(T_{f t}-\left(T_{f s}+T_{f m}\right)\right)^{2}
$$

The non gradient based complex method Box (1965)Krus et al. (1991) was used with a population of 36 designs. For every iteration in the optimization routine, the population is adjusted by taking the worst design and mirror it with a factor of 1.3 across the center of gravity of the population. Table 3 shows the optimized parameters for the crane investigated in this paper (Crane 2) and previous results from a similar crane (Crane 1) investigated in a previous paper, Mgslätt and Hansen (2018).

the optimization routine showed no need for a term representing Coulomb friction, $C_{2}$, hence the overall system model is simplified to:

$$
\begin{array}{r}
T_{f s}=\left(C_{1} \cdot T_{d}+C_{3}\right) \cdot e^{\frac{-\left|\omega_{m}\right| \cdot 60}{C_{4} \cdot 2 \pi}} \\
+C_{5} \cdot \omega_{m}+C_{6} \cdot T_{d}
\end{array}
$$

Figure 8 shows how the distribution between Coulomb, stiction, and viscous friction is within the system friction model for Crane 1 and Crane 2. The
Table 3: Optimized system friction parameters

\begin{tabular}{|l|c|c|}
\hline Model & $\begin{array}{c}\mathbf{1 5 0} \text { t crane } \\
\text { Crane } \mathbf{1}\end{array}$ & $\begin{array}{c}\mathbf{1 5 0} \text { t crane } \\
\text { Crane 2 }\end{array}$ \\
\hline $\mathbf{C}_{\mathbf{1}}[-]$ & $328 \cdot 10^{-7}$ & $155 \cdot 10^{-7}$ \\
\hline $\mathbf{C}_{\mathbf{2}}[\mathrm{Nm}]$ & 0.0 & 0.0 \\
\hline $\mathbf{C}_{\mathbf{3}}[\mathrm{Nm}]$ & 3.15 & 0.562 \\
\hline $\mathbf{C}_{\mathbf{4}}\left[\frac{\mathrm{rev}}{\mathrm{min}}\right]$ & 157 & 229 \\
\hline $\mathbf{C}_{\mathbf{5}}[\mathrm{Nm} \cdot \mathrm{s}]$ & 0.00851 & 0.00943 \\
\hline $\mathbf{C}_{\mathbf{6}}[-]$ & $121 \cdot 10^{-7}$ & $162 \cdot 10^{-7}$ \\
\hline$E$ & $N A$ & 1345 \\
\hline
\end{tabular}

friction is estimated based on both cranes having a hanging load of $100 \mathrm{t}$ of a maximum $150 \mathrm{t}$. The calculated system friction is shown as the torque applied on each motor shaft (Crane 1 and Crane 2 have a total of 11 motors).

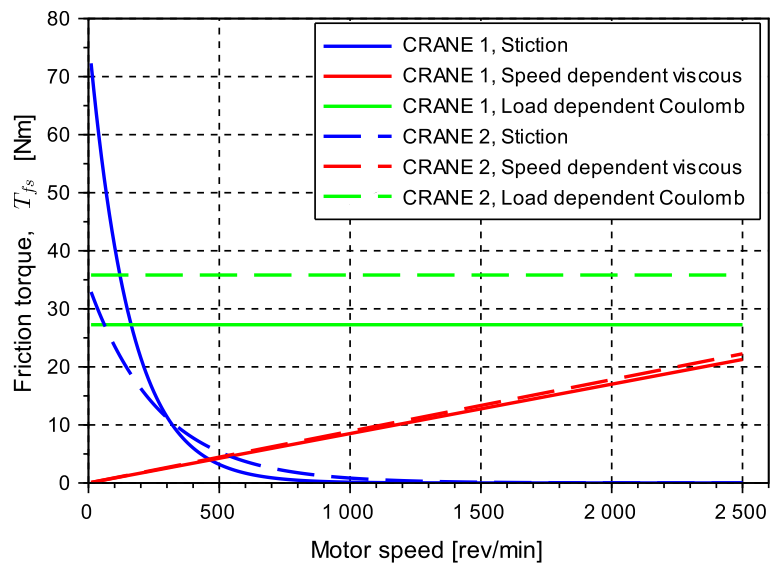

Figure 8: Comparison of modeled friction in Crane 1 and Crane 2. Crane load is $100 \mathrm{t}$.

\section{Model modifications}

One of the benefits of using parametric models is that the parameters can be modified to better fit the experimental data from the system investigated. The method up til now has been to define hydraulic motor parameters based on sub-supplier data, then defining system friction parameters based on measured results (ref. first and second box in Fig. 9). To see if results can be further improved a simple iterative optimization is performed (see Fig. 9). An additional loop of optimizing the model parameters is added were the system friction parameters were locked and the motor friction parameters opened, and this time optimized 


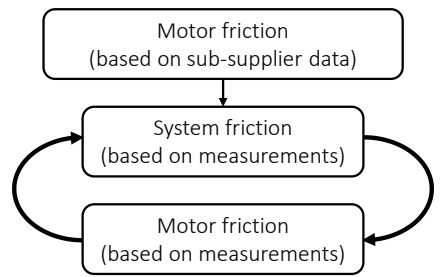

Figure 9: Iterative optimization loop of friction parameters.

based on the measured results instead of data from the sub-supplier. Next the motor model parameters will be locked again and new system friction parameters will be determined. In principle, this could be performed in several loops, however, results revealed that significant improvements were only obtained in the first loop.

Table 4: Optimized friction parameters

\begin{tabular}{|l|c|c|}
\hline Model & $\begin{array}{c}\text { Crane 2, } \\
\text { first loop }\end{array}$ & $\begin{array}{c}\text { Crane 2, } \\
\text { second loop }\end{array}$ \\
\hline $\mathbf{K}_{\mathbf{1}}[\mathrm{Nm} \cdot \mathrm{s}]$ & $4.05 \cdot 10^{-3}$ & $0.0 \cdot 10^{-3}$ \\
\hline $\mathbf{K}_{\mathbf{2}}\left[\frac{\mathrm{N} \cdot \mathrm{s}^{2}}{\mathrm{~m}^{8}}\right]$ & $3.69 \cdot 10^{10}$ & $4.5 \cdot 10^{10}$ \\
\hline $\mathbf{K}_{\mathbf{3}}[-]$ & 0.0050 & 0.012 \\
\hline $\mathbf{K}_{\mathbf{4}}[\mathrm{Nm}]$ & 14.0 & 12.8 \\
\hline $\mathbf{K}_{\mathbf{5}}\left[\frac{\mathrm{m}^{5}}{\mathrm{~N}}\right]$ & $1.16 \cdot 10^{-14}$ & $0.0 \cdot 10^{-14}$ \\
\hline $\mathbf{C}_{\mathbf{1}}[-]$ & $155 \cdot 10^{-7}$ & $141 \cdot 10^{-7}$ \\
\hline $\mathbf{C}_{\mathbf{2}}[\mathrm{Nm}]$ & 0.0 & 0.0 \\
\hline $\mathbf{C}_{\mathbf{3}}[\mathrm{Nm}]$ & 0.562 & 2.38 \\
\hline $\mathbf{C}_{\mathbf{4}}\left[\frac{\mathrm{rev}}{\mathrm{min}}\right]$ & 229 & 270 \\
\hline $\mathbf{C}_{\mathbf{5}}[\mathrm{Nm} \cdot \mathrm{s}]$ & 0.00943 & 0.0089 \\
\hline $\mathbf{C}_{\mathbf{6}}[-]$ & $162 \cdot 10^{-7}$ & $159 \cdot 10^{-7}$ \\
\hline$E$ & 1345 & 938 \\
\hline
\end{tabular}

Doing the extra step of optimization affects the deviation between simulated and measured results used for optimization. This can be seen from the cost function, E, which is reduced by $30 \%$ (4). From the changes, it is observed that viscous losses $\left(K_{1}=0\right)$ and highpressure losses $\left(K_{5}=0\right)$ in motor friction have been practically eliminated, but instead, the parameter for turbulent losses has increased $\left(K_{2}\right.$ parameter increased with $22 \%$ ). In the system friction model, there are only smaller changes except for the $C_{3}$ parameter, which has quadrupled in size. However, even if the increase is relatively significant, the added $2 \mathrm{Nm}$ does not significantly impact the overall stiction loss.

When looking at the motor model changes in Fig. 10, it can be seen that the turbulent losses are increased. This is seen by the increase in high speed, high dis-

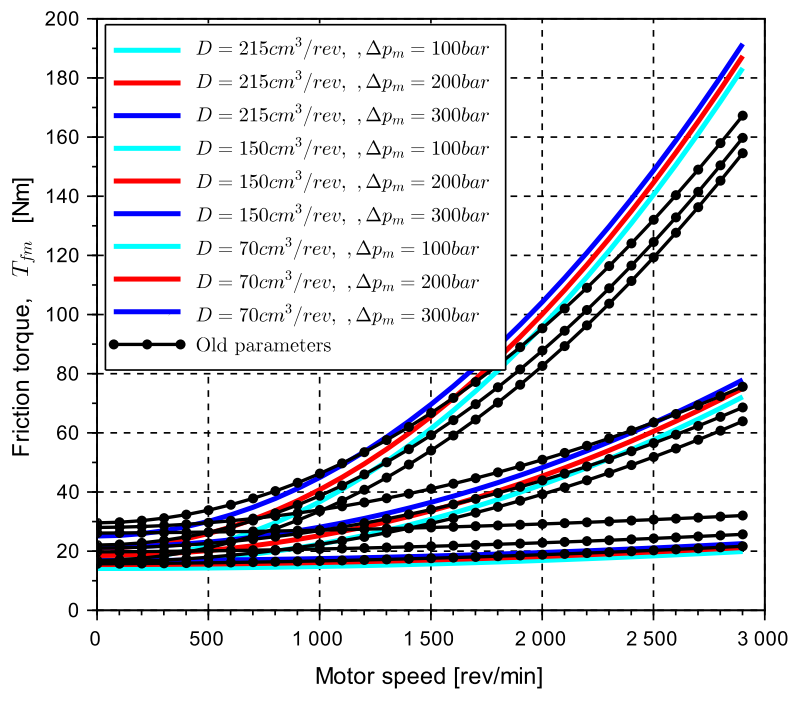

Figure 10: New motor parameters compared to old.

placement friction. The modification has also led to a significant decrease in friction at low displacements. It has to be taken into consideration that the test scenarios used for the optimization of parameters do not include displacement settings lower than $160 \mathrm{~cm}^{3} / \mathrm{rev}$.

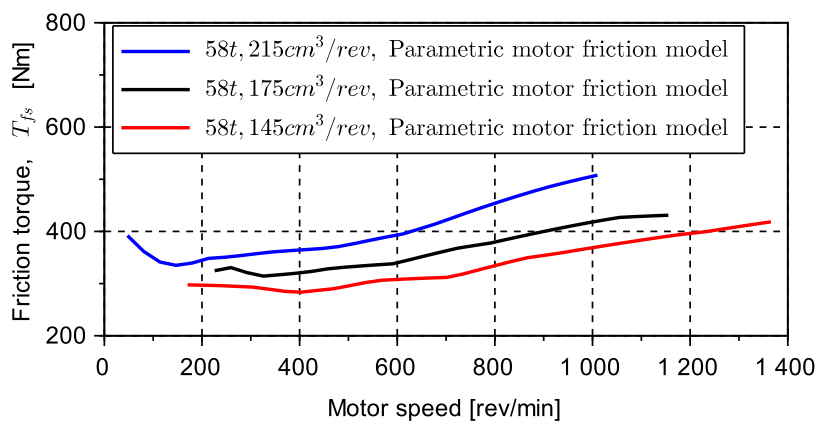

Figure 11: System friction derived from measurements.

An observation when analyzing the measurements is that similar working conditions for the mechanical system result in different values of the calculated system friction. An example is when the winch is operated with the same load at the same speed, but with different motor displacement. With a perfect motor friction model, these values should be the same, and the curves in Fig. 11 should be coincident. The estimation difference at $1000 \mathrm{rev} / \mathrm{min}$ is approximately $100 \mathrm{Nm}$, when comparing the 145 and $215 \mathrm{~cm}^{3} / \mathrm{rev}$ displacement setting (see Fig. 11). The estimation error indicates that there are unmodeled friction losses in the hydraulic motors.

The additional set of tests with $107 \mathrm{t}$ and $77 \mathrm{t}$ (see Fig. 12) show the same tendencies as seen for the 58 


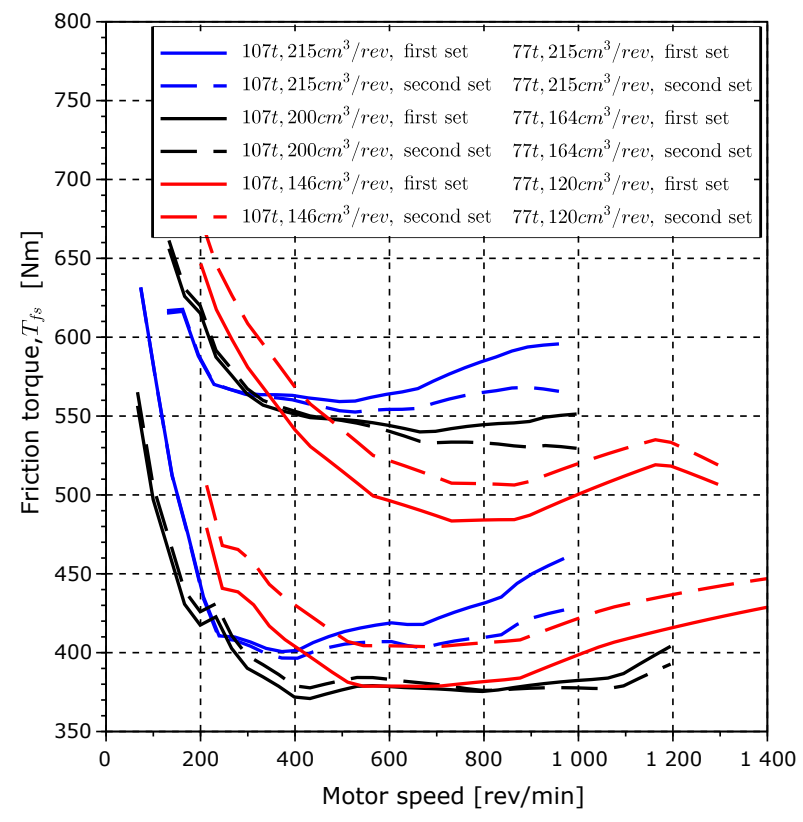

Figure 12: Calculated total system friction for tests with 77 and 107t load.

t test in Fig. 11, indicating that there are unmodeled friction losses in the hydraulic motors. A solution to this problem could be introducing a second optimization loop and modifying the motor friction model based on the measurements. In Fig. 12, the system friction is derived from measurements using both the first optimization loop and the second. The results are slightly better, but the deviation is still approximately $100 \mathrm{Nm}$, indicating that there are variations that the model cannot cover. The unmodeled friction is not a result of the parametric model's ability to represent the measurements from the sub-supplier. This is shown in Fig. 13, where the system friction is derived based on a blackbox (map) friction model of the motors. The black-box model is based on the sub-suppliers efficiency map, using linear interpolation. The use of the black-box does not show any significant improvement, and at low loads (Test 1 and Test 12), the parametric model is significantly better. Overall it must be expected that the sub-supplier data will not necessarily be transferable to other situations.

The effect of a second optimization loop was also checked for the low load scenario with $0 \mathrm{t}$ hook load (Fig. 14). The results are not particularly improved with the second optimization loop. This is not unexpected since low load scenarios are not highly prioritized in the second optimization loop. Unlike the other load cases, this one shows quite small variations of the estimated system friction, which indicates that the motor model is working quite well under these conditions.

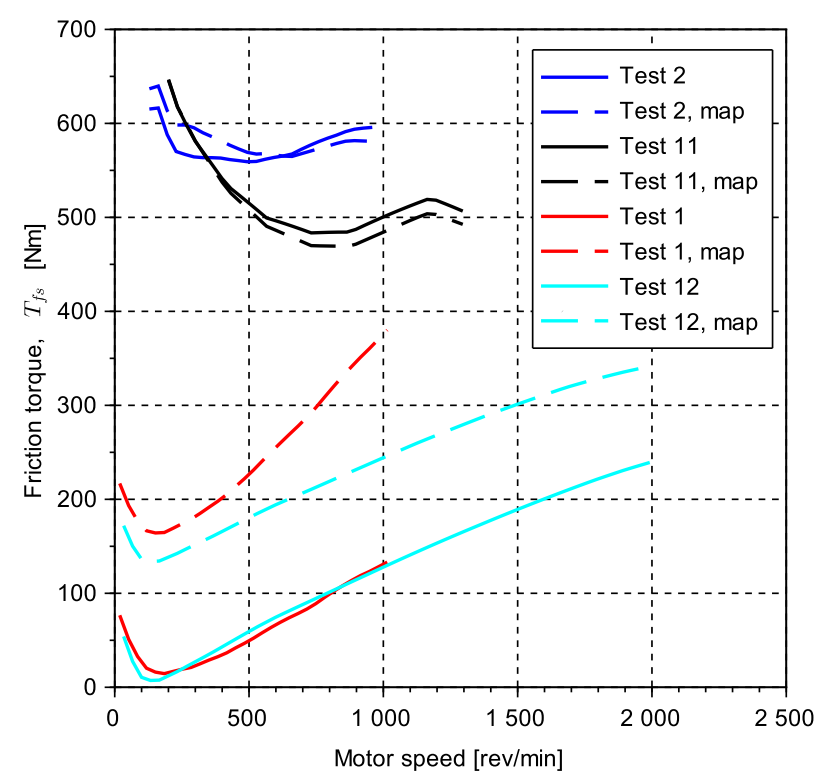

Figure 13: System friction calculated based on model versus efficiency map from supplier.

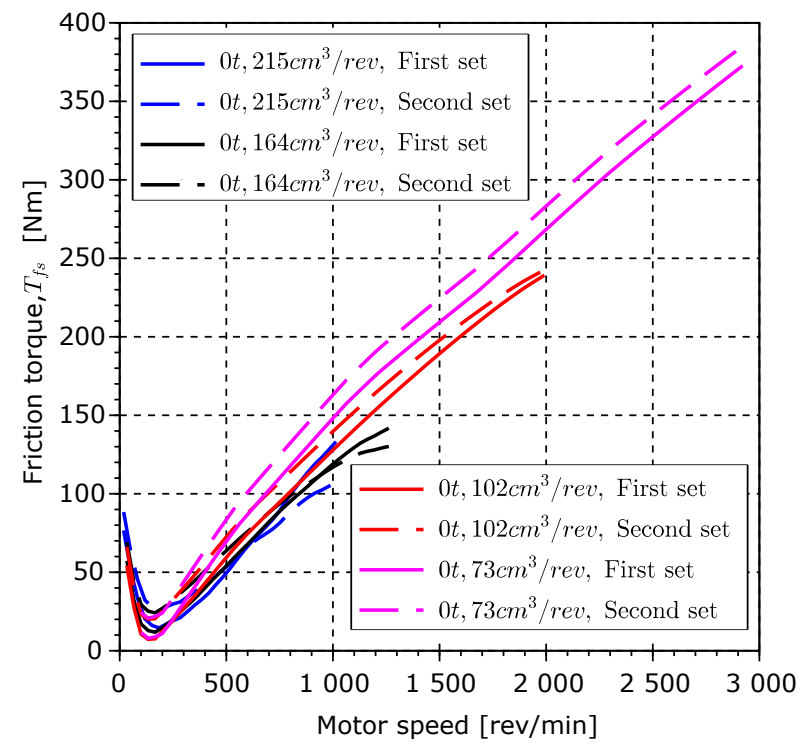

Figure 14: Calculated system friction for tests with $0 \mathrm{t}$ hook load (hook weighs approx 2t).

The two parameter-sets were also compared in a simulated AHC scenario replicating the real test mentioned in section 4 (see Fig. 15). Each active side motor is set to $215 \mathrm{~cm}^{3} / \mathrm{rev}$, and the winch load is $55 \mathrm{t}$ on the outer wire layer.

The implementation of a second optimization loop did not show any significant improvement of the results when looking at the test done with simulated waves and activated AHC (Fig. 15). During the op- 


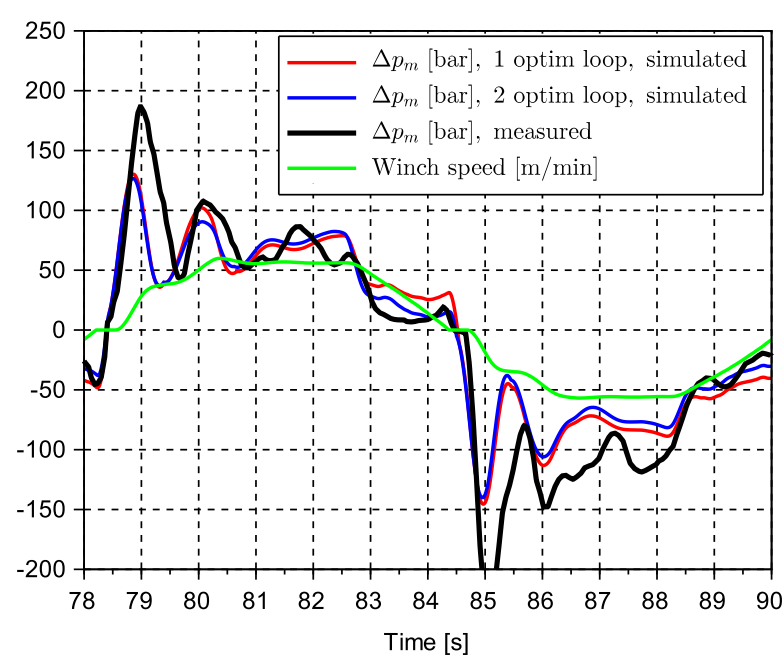

Figure 15: Measured and simulated pressure drop.

timization, the cost was significantly reduced, which should comply with better results. An explanation for not getting this improvement is assumed to be that the measurements, which are used as a reference for the second optimization of motor model parameters and the system friction parameters, do not include any low displacement settings. In the verification test performed, the eight passive motors are set to $70 \mathrm{~cm}^{3} / \mathrm{rev}$ and the three active motors are set to $215 \mathrm{~cm}^{3} /$ rev.

Conclusions are difficult to extract from these results, but previously performed tests on similar systems have had the same tendency that the calculated system friction differs when the motors' displacement is changed (ref. Crane 1, which was investigated in Q1 2018 Moslàtt and Hansen (2018)). The deviation is slightly decreased with the second optimization loop, and for the displacement changes shown in Fig. 12, the deviation is less than $10 \%$ from the average value. The results indicate that motor friction has some additional losses dependent on displacement and probably also some system-dependent variables like oil properties and working temperature since these losses are not captured in the sub-supplier data. The overall friction is not affected too much since the system friction model is anyway fitted to the remaining friction. The deviation is not insignificant, but it has to be considered that the system friction will have to cover this deviation, leading to a nonperfect distribution between motor friction and system friction.

\section{Verification}

The verification process was divided into three parts:

- Checking deviation between the measured data and the friction model.

- Checking the actual difference in pressure drop in a simulation model vs. measurements (normal winch mode).

- Checking the actual difference in pressure drop in a simulation model vs. measurements (Active heave compensated winch mode).

\subsection{Measured vs. modeled friction}

Three further tests (Test 8, 9, and 10), see Table 5, were added and combined with tests 2,3 , and 5 from Table 2, yielding a set of high load tests. The high load scenarios are prioritized because they most often are the critical design parameter for system design.

Table 5: Evaluated test scenarios

\begin{tabular}{l|l|l}
\hline Name & $\begin{array}{l}\text { Motor } \\
\text { displacement } \\
\text { (total/single) }\end{array}$ & Winch load \\
\hline Test 8 & $2200 / 200 \mathrm{~cm}^{3} / \mathrm{rev}$ & $107 \mathrm{t}$ \\
Test 9 & $1600 / 146 \mathrm{~cm}^{3} / \mathrm{rev}$ & $77 \mathrm{t}$ \\
Test 10 & $1320 / 120 \mathrm{~cm}^{3} / \mathrm{rev}$ & $77 \mathrm{t}$ \\
Test 11 & $1600 / 146 \mathrm{~cm}^{3} / \mathrm{rev}$ & $107 \mathrm{t}$ \\
Test 12 & $1120 / 102 \mathrm{~cm}^{3} / \mathrm{rev}$ & $0 \mathrm{t}$ \\
\hline
\end{tabular}

Figure 16 shows a comparison between the modeled total winch system friction versus the measured. The torque is referred to the motor shaft but represents the total loss of all 11 motors and associated components. The results shown in Fig. 16 look promising, and the maximum deviation is approximately $50 \mathrm{Nm}$. In general, there is a good correlation between the model and measurements on both the shape as well as the percentage deviation. However, the low speed below $200 \mathrm{rev} / \mathrm{min}$ is more inaccurate, which can be seen in Fig. 17, where the deviation between the results (error) is shown in percentage of the nominal system torque.

\subsection{Normal winch mode scenario}

The test is executed in what is called normal mode, which is a manually operated mode where the crane operator controls the winch with the joystick. A simulation model of the winch system was developed in the commercial modeling and simulation software Simulation $\mathrm{X}^{\mathrm{TM}}$. For example, the measured and simulated pressure drop across the motors are shown for Test 3 in Fig. 18. The results shows a good correlation between estimated pressure in the model and real measured values from the tests. However, the results also indicate that the friction model underestimates 


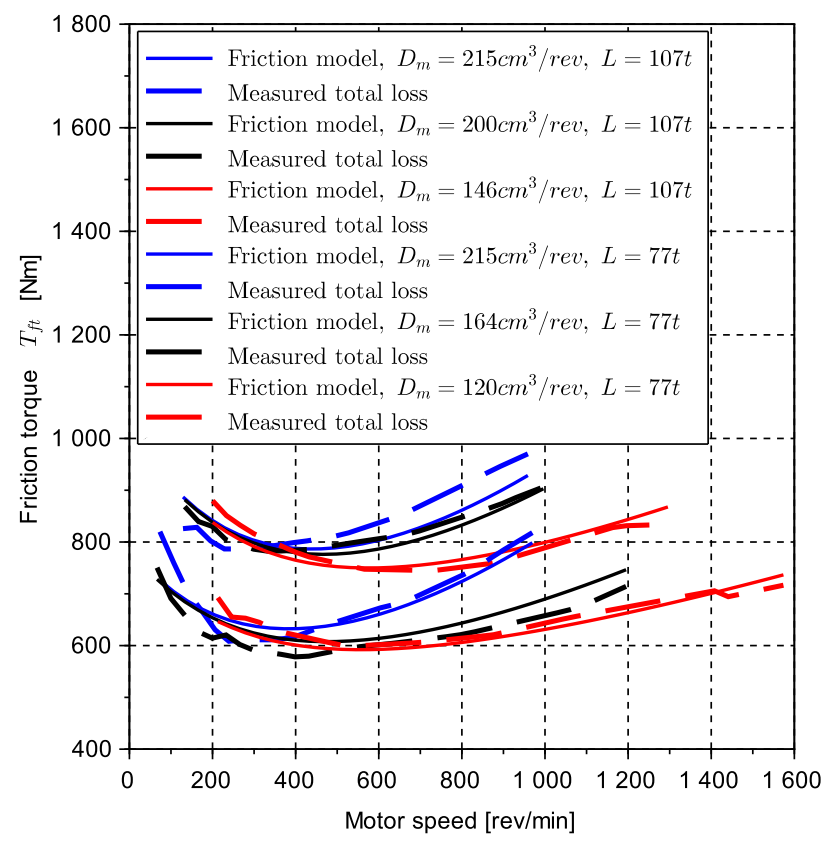

Figure 16: Compared model versus measured results.

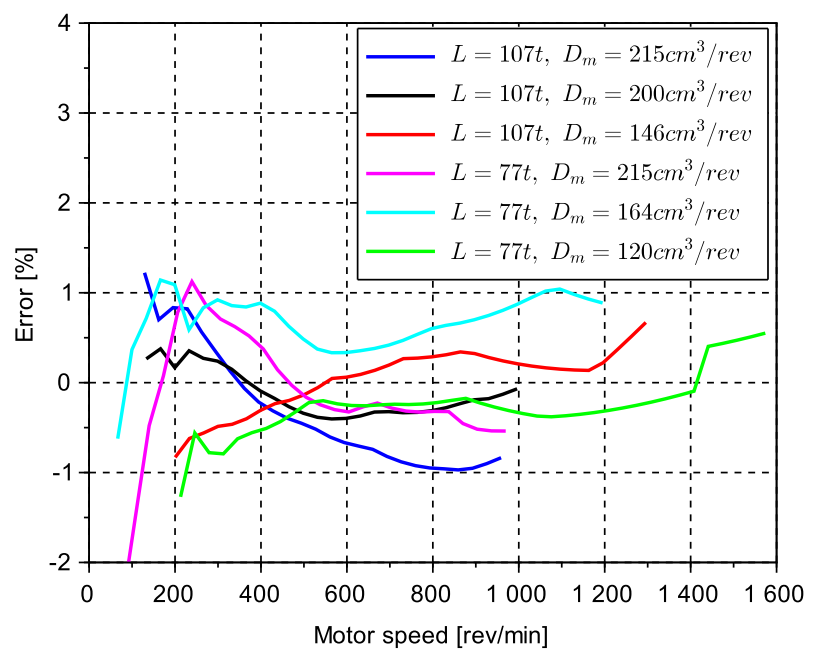

Figure 17: Model error in percentage of the nominal system torque.

the losses when the displacement setting is low, and the speed is high.

\subsection{AHC scenario}

As a third step of verifying the model, it is of interest to look at a situation in AHC. For that purpose, a specially designed onshore test had to be conducted without the actual heave motion but still moving the load in reference to an artificially generated wave mo-

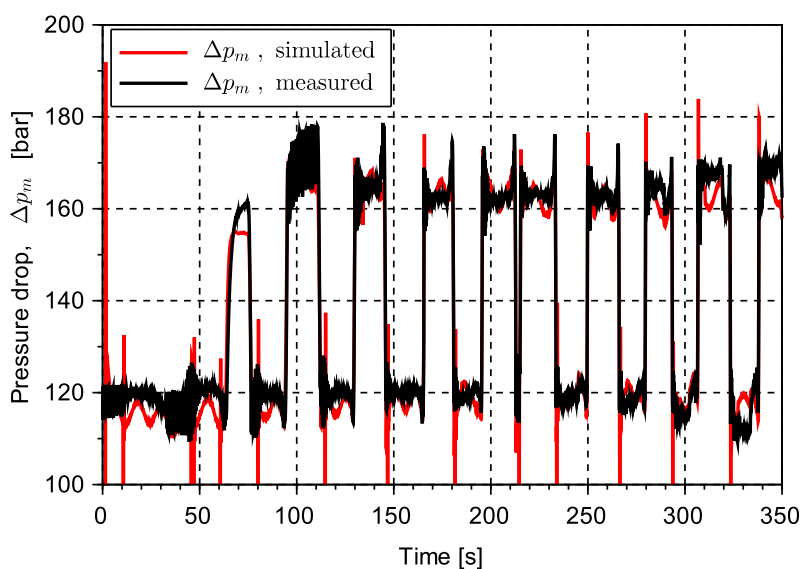

Figure 18: Comparison of measured versus simulated pressure drop across the motors for Test 3.

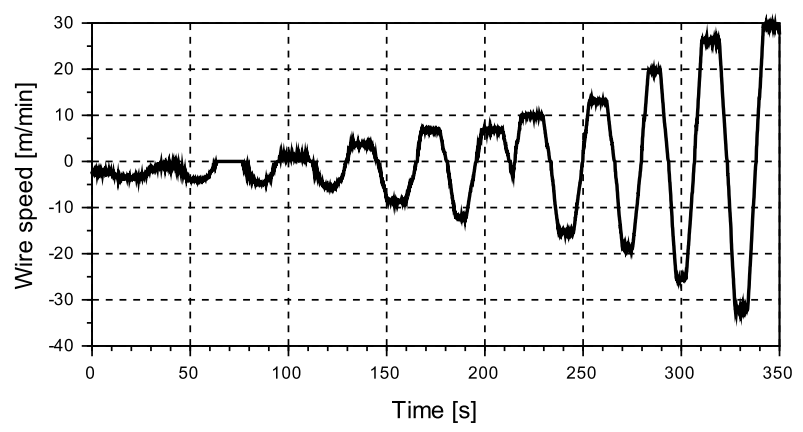

Figure 19: Winch speed profile for the test results shown in Fig. 18

tion. An important thing to notice is that when the winch system is configured for AHC, all friction is absorbed by the active side motors. This friction includes friction generated from both the passive and the active side of the winch system. The passive side will not have the same displacement setting and pressure levels as in the active loop. Thus the total friction will reflect a combination of different motor displacements and pressures.

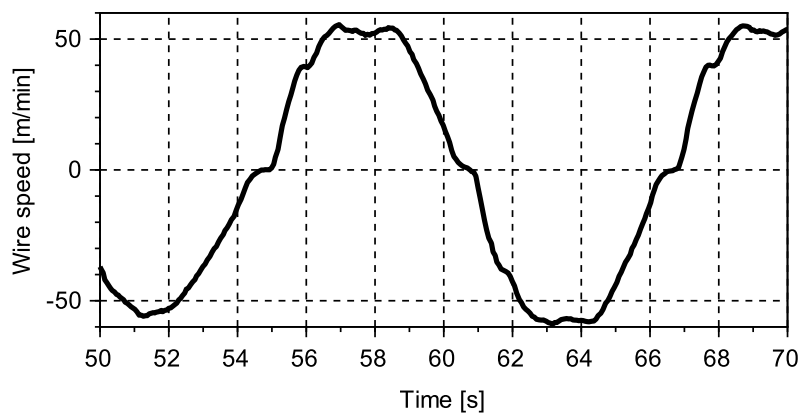

Figure 20: Winch speed profile for Fig. 21 


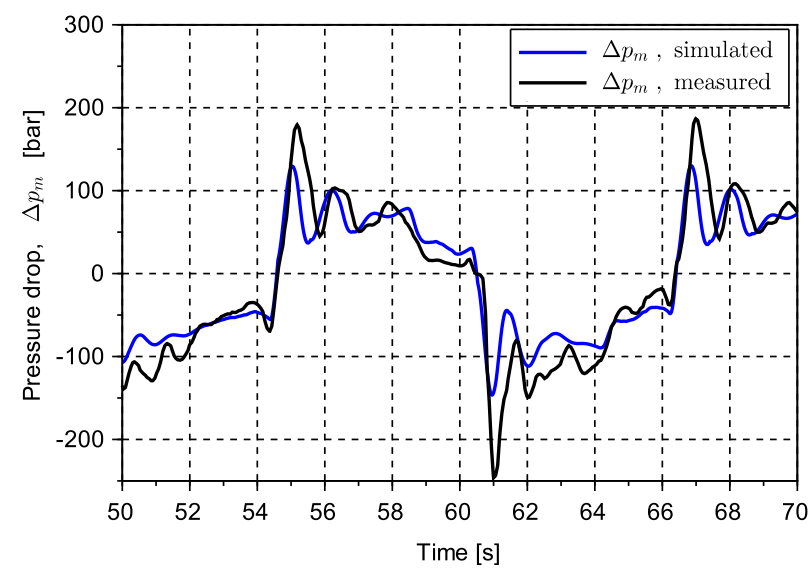

Figure 21: Measured and simulated pressure drop across the motors (active side), with winch speed according to Fig. 20.

In Fig. 21, the winch is operated in $\mathrm{AHC}$ at the outer layer with an attached load of $55 \mathrm{t}$. The active side motors are set to $215 \mathrm{~cm}^{3} / \mathrm{rev}$. The pressure estimated by simulations shows a decent accuracy. When paying out wire, the error is approximately 20 bar, and when pulling in, the error is significantly lower and close to zero. This implies an overall deviation of 10 bar of the actual 130 bar. In the next test scenario (Fig. 22), the motor displacement on the three active motors is set to $160 \mathrm{~cm}^{3} / \mathrm{rev}$, and the load is kept the same.

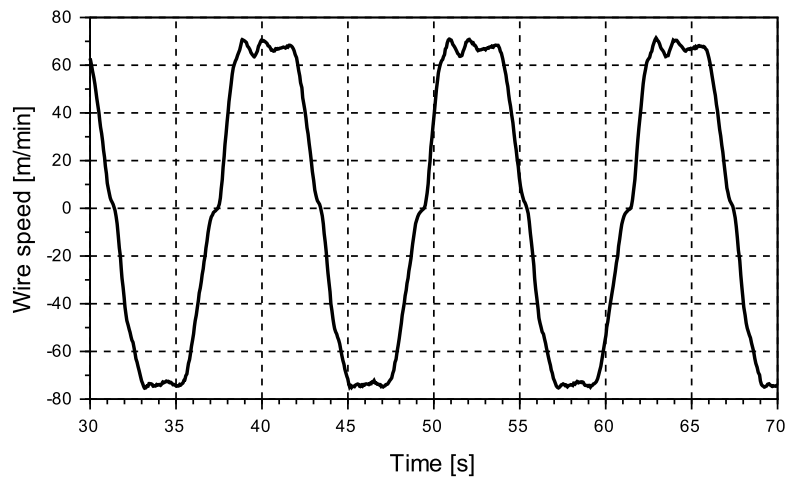

Figure 22: Winch speed profile for Fig. 23

Results are shown in Fig. 23 show about the same or better accuracy as the results with $215 \mathrm{~cm}^{3} / \mathrm{rev}$, with a pressure deviation of \pm 10 bar at the areas constant velocity (low-pressure disturbance by load dynamics). The passive side is in both scenarios in Fig. 21 and 23 , pressurized with a more or less constant pressure drop across the motors of approximately 275 bar. The displacement is balancing the load at approximately 70 $\mathrm{cm}^{3} / \mathrm{rev}$ per motor. For these two scenarios, a 10 bar deviation gives a resulting 3 and $4 \%\left(160 \mathrm{~cm}^{3} / \mathrm{rev}\right.$ and

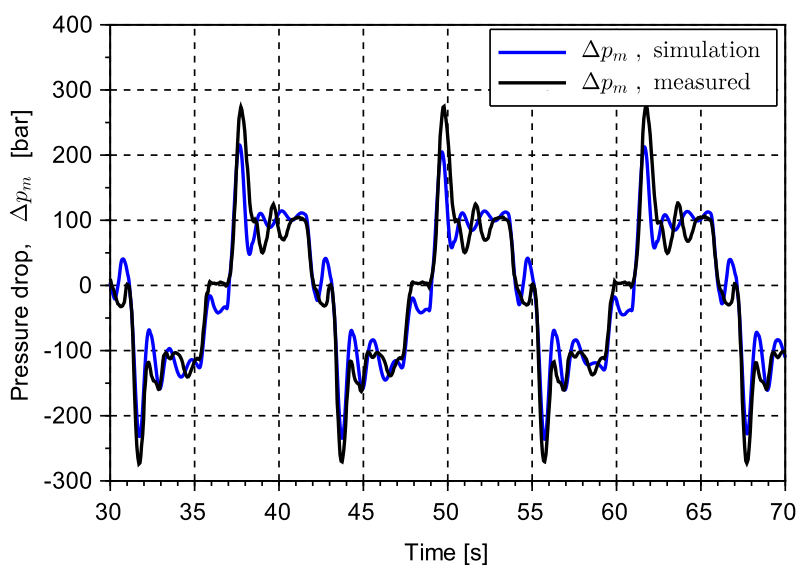

Figure 23: Measured and simulated pressure drop across the motors (active side), with winch speed according to Fig. 22.

$215 \mathrm{~cm}^{3} / \mathrm{rev}$ ) error in the estimated loss (in percentage of the nominal winch torque). This is more than what was measured at the previous tests in normal winch mode, where the results gave up to $1 \%$ error.

\section{Conclusions}

The method for friction estimation shows friction estimation results within $1 \%$ of the nominal load at speeds higher than $250 \mathrm{rev} / \mathrm{min}$, which is acceptable. An additional test to verify the model in an active/passive heave compensated setup was performed in AHC with a $55 \mathrm{t}$ winch load. The model gave an error average of 10 bar giving a relative error of approximately $10 \%$ of the measured pressure. The verification test was done with a $55 \mathrm{t}$ load on a crane designed for a maximum of 150 t. Tests with higher loads would be preferred, but unfortunately not possible at this point. Despite this, the confidence in the model is quite good since the model structure is divided into two parts. One for the hydraulic axial piston motors and one for the remaining mechanical system. The motor model was made by the use of sub-supplier datasheets and covered a large part of the motors operating conditions with respect to displacement settings, pressure, and speed. The second part of the model was estimated based on several tests done on the full-size crane. The tests were done with a wide spectrum of loads, speeds, and displacement settings. The loads in these tests reached a maximum at $107 \mathrm{t}$, which is over $70 \%$ of the maximum safe working load (SWL). An additional test to see if the modification/optimization of the motor friction model parameters could improve the overall results was looked into. A second optimization loop was added to improve the model parameters for motor friction based on the real 
measurements done on the crane (not by using the subsupplier data). The second optimization loop resulted in an improved result of the cost function. It was seen that the new set of parameters gave a more realistic estimation of the system friction (based on measurements and motor friction model), which indicates an improved motor friction model. Although the model parameters seem to be improved, it leaves some uncertainty about the complete range of the model with respect to load and motor displacement. The second optimization loop is likely to give a better distribution between motor friction and system friction, but the uncertainty increases due to a limited range of data to use in the optimization routine. The improvement is shown to be most significant at high loads (see fig. 12).

\section{References}

Bosch Rexroth AG. Sales Information, Axial Piston Units. 2010.

Box, M. J. A New Method of Constrained Optimization and a Comparison With Other Methods. The Computer Journal, 1965. 8(1):42-52. doi:10.1093/comjnl/8.1.42.

Huhtala, K. and Villenius, M. Comparison of SteadyState Models of Hydraulic Pump. 1997.

Jeong, H. S. and Kim, H. E. A novel performance model given by the physical dimensions of hydraulic axial piston motors: Experimental analysis. Journal of Mechanical Science and Technology, 2007. 21(4):630-641. doi:10.1007/BF03026968.
Krus, P., Jansson, A., and Palmberg, J.-O. Optimization for Component Selection Hydraulic Systems. In Fourth Bath International Fluid Power Workshop. 1991.

Moslått, G.-A. and Hansen, M. R. Modeling of Friction Losses in Offshore Knuckle Boom Crane Winch System. In 2018 Global Fluid Power Society PhD Symposium, GFPS 2018. IEEE, pages 1-7, 2018. doi:10.1109/GFPS.2018.8472316.

Moslått, G.-A., Hansen, M. R., and Karlsen, N. S. A model for torque losses in variable displacement axial piston motors. Modeling, Identification and Control, 2018. 39(2):107-114. doi:10.4173/mic.2018.2.5.

Ortwig, H. New Method of Numerical Calculation of Losses and Efficiencies in Hydrostatic Power Transmissions. SAE International Off-Highway Congress, 2002. 1418(724). doi:0148-7191.

Pacey, D. A., Turnquist, R. O., and Clark, S. J. The development of a coefficient model for hydrostatic transmissions. In In: Proc.35th Nat.conf.on Fluid Power, volume 33. 35th National Conference in Fluid Power, Chicago, pages 173-178, 1979.

Schlösser, W. Mathematical model for displacement pumps and motors, part 1. Hydraulic Power Transmission, 1961. pages 252-269.

Wilson, W. Performance criteria for positive displacement pumps and fluid motors. In ASME Semiannual Meeting, paper No. 48-SA-14. 1948. 